\title{
Análisis de la movilidad de pesticidas en los suelos de la región centro-sur del estado de Chihuahua
}

Analysis of pesticide mobility in the soils of the central-south region of the state of Chihuahua

\author{
Vicente Moncayo-Cassiano $^{1}$, Bertha Alicia Rivas Lucero ${ }^{1 凶}$, Sergio Guerrero Morales1, Gabriel Zúñiga Avila ${ }^{1}$ y \\ José Eduardo Magaña Magaña ${ }^{1}$. \\ ${ }^{1}$ Universidad Autónoma de Chihuahua-Facultad de Ciencias Agrícolas y Forestales, Km 2 1⁄2 Carretera Delicias- \\ Rosales C.P. 33000, Tel: 6394722726 \\ ${ }^{\square}$ Autor para correspondencia: brivas@uach.mx.
}

Recibido: 15/07/2017

Aceptado: 10/12/2017

\section{RESUMEN}

Los pesticidas son ampliamente usados en la producción de alimentos en la región Centro-Sur del Estado de Chihuahua perteneciente al Distrito de Riego 005. La caracterización del destino final y la toxicidad de estos son importantes para evaluar con certeza el riesgo asociado a su uso. Esta investigación se enfocó en el análisis del metabolismo y residuos de los principales insecticidas utilizados para el control de plagas en los cultivos de mayor importancia en esta región, tomando en cuenta los tipos de suelos para determinar su posible migración a los cuerpos de agua o su permanencia en el terreno lo cual permite comprender el problema de la contaminación por pesticidas en esta región y así contribuir al uso y manejo adecuado de estos. Los resultados muestran que los principales pesticidas usados en este Distrito son esencialmente organofosforados de los cuales en su mayoría son considerados muy tóxicos y el resto tóxicos y nocivos. Así mismo, el análisis muestra que muchos de estos no son persistentes en el ambiente. Sin embargo, su uso intensivo es lo que puede ocasionar peligros ambientales a corto y largo plazo. Las interacciones entre los plaguicidas aplicados y el ambiente, dependen fundamentalmente de las propiedades de los plaguicidas así como de los factores ambientales, por lo cual es importante que los productores consideren el tipo de plaguicida a aplicar, características del suelo, la dosis recomendada y tener en cuenta que las buenas prácticas de manejo de estos puede reducir el riesgo de contaminación en la región.

Palabras clave: Pesticidas, Movilidad, Suelos.

\begin{abstract}
ABSTRAC
Pesticides are widely used in food production at the Central-South region of the State of Chihuahua belonging to the District of Irrigation 005. The characterization of the final destiny and the toxicity of these are important to evaluate with certainty the risk associated with its use. This research focused on the analysis of the metabolism and residues of the main insecticides used for the pest control in the most important crops in this region, taking into account the types of soils to determine their possible migration to water bodies or their permanence in the land which allows to understand the problem of pesticide
\end{abstract}


contamination in this region and thus contribute to the proper use and management of these. The results show that the main pesticides used in this District are essentially organophosphorus, of which most are considered very toxic and the rest are toxic and harmful. Also, the analysis shows that many of these are not persistent in the environment. However, its intensive use is what can cause environmental hazards in the short and long term. The interactions between the applied pesticides and the environment depend fundamentally on the pesticides properties as well as the environmental factors, which it is important that the producers consider the type of pesticide to apply, soil characteristics, the recommended dose and note that good management practices can reduce the risk of contamination in the region.

Keywords: Pesticides, Movility, Soils.

\section{INTRODUCCIÓN}

Los pesticidas son ampliamente usados en la producción de alimentos y se cree que existen más de 1000 tipos de pesticidas en uso. Muchos de estos son usados en grandes cantidades en la Región Centro-Sur del Estado de Chihuahua. Estos contaminantes permanecen en el suelo un largo tiempo después de la aplicación, e ingresan en los cursos de agua, incorporándose a las cadenas alimenticias (Dokic, M, et. al., 2012). El efecto de los pesticidas sobre el terreno sembrado se expande hacia el aire y se depositan en el agua, contaminando las aguas subterráneas, los ríos y lagos, así como los alimentos cultivados en terrenos donde se utilizó. Estudios en el ámbito internacional revelan un grave daño en el ambiente, sobre todo daños a la salud del hombre en zonas expuestas al impacto de estos productos.

Se estima que sólo un 0.1 por ciento de la cantidad de plaguicidas aplicados llega a la plaga, mientras que el restante circula en el ambiente, contaminando el suelo, agua y la biota; por lo tanto, es importante caracterizar el destino y la toxicidad no prevista de estos plaguicidas para evaluar con certeza el riesgo asociado a su uso (Carvalho et al, 1998). Esta investigación se enfocó en el análisis del metabolismo y residuos de los insecticidas utilizados en los principales cultivos de la región Centro-Sur del Estado de Chihuahua en el suelo y su posible migración en el ambiente, lo que permitirá comprender el problema y así contribuir al uso y manejo adecuado de estos. Lo anterior con la finalidad de evitar residualidad en los suelos e impedir contaminar los cuerpos de agua y por consiguiente evitar la afectación de la salud de la población y de los ecosistemas acuáticos. Finalmente, el estudio propone posibles estrategias para el uso de los pesticidas de tal manera que no se conviertan en zonas de riesgo.

\section{MATERIALES Y MÉTODOS}

1. Lugar.- El estudio se realizó en la región Centro-Sur del Estado de Chihuahua.

2. Fuentes de información.- Se determinó la superficie sembrada de los principales cultivos de interés en la región, así como los tipos de suelo en base a las características de los perfiles de suelo.

Así mismo se determinó tipos y dosis por ha de insecticidas usados para el control de plagas en los principales cultivos, analizandose el metabolismo y residuos de estos para determinar los riesgos hacia el medio ambiente (De Liñan, 2003).

3. Análisis de información.- Se integraron las fuentes de información para determinar tipos de insecticidas, cantidades usadas, características y riesgos en el ambiente.

\section{RESULTADOS}

Suelos del Distrito de Riego 005 de la Región Centro-Sur del Estado de Chihuahua

Revista Científica Biológico Agropecuaria Tuxpan 5 (2) 
En la Figura 1 se observa el plano de suelos del Distrito de Riego 005 los cuales son diferenciados por colores y su descripción se muestra en los perfiles de la parte izquierda superior cuyos detalles se observan en la Figura 2. En estos detalles, se observa que los suelos que predominan son los migajones arenosos representados por las series Jaquez, Bachimba,
Ortiz, Rosales, Loreto, Cuadra, Snyman y Armendáriz; seguidos por los suelos francos (Series Dolores, Conchos y Colomina), migajón arcilloso (Series González, Galeana y Consuelo) y suelos arenosos (Series Meoqui y Delicias). Estos se caracterizan por su escasa materia orgánica.

Figura 1. Mapa de suelos del Distrito de Riego 005 con detalle de perfiles (CNA Delicias, 2013).

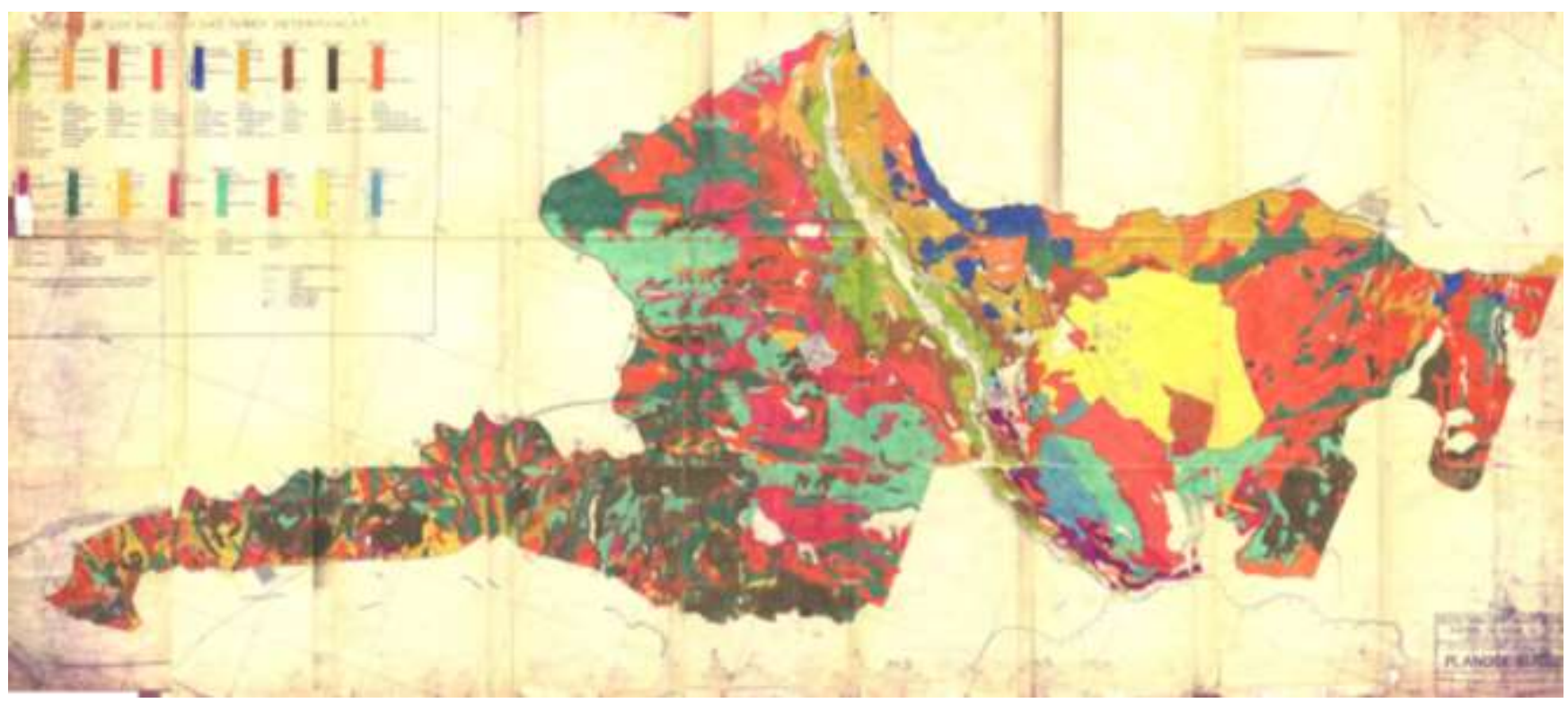

Figura 2. Perfiles de los suelos en las series determinadas
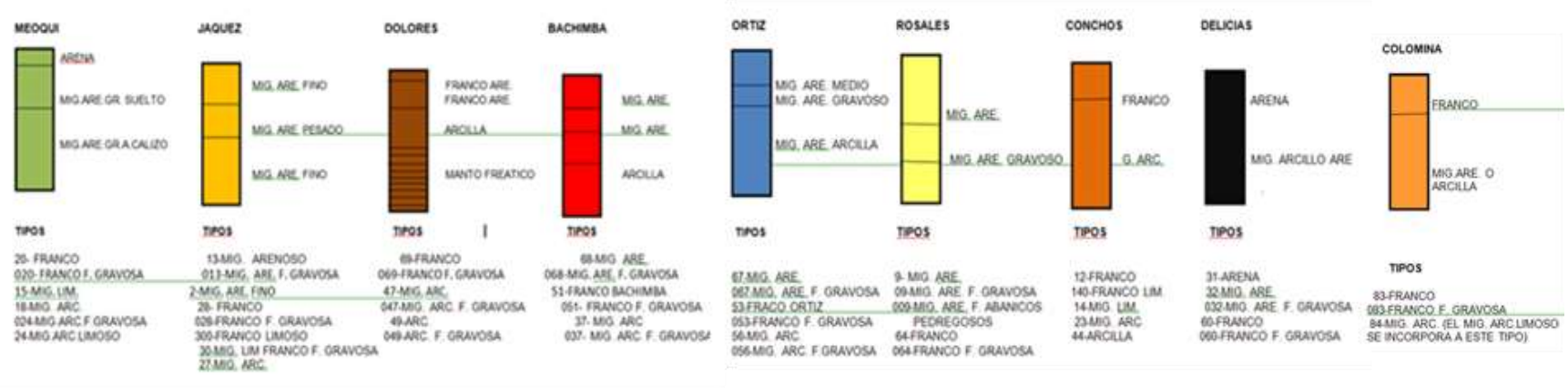


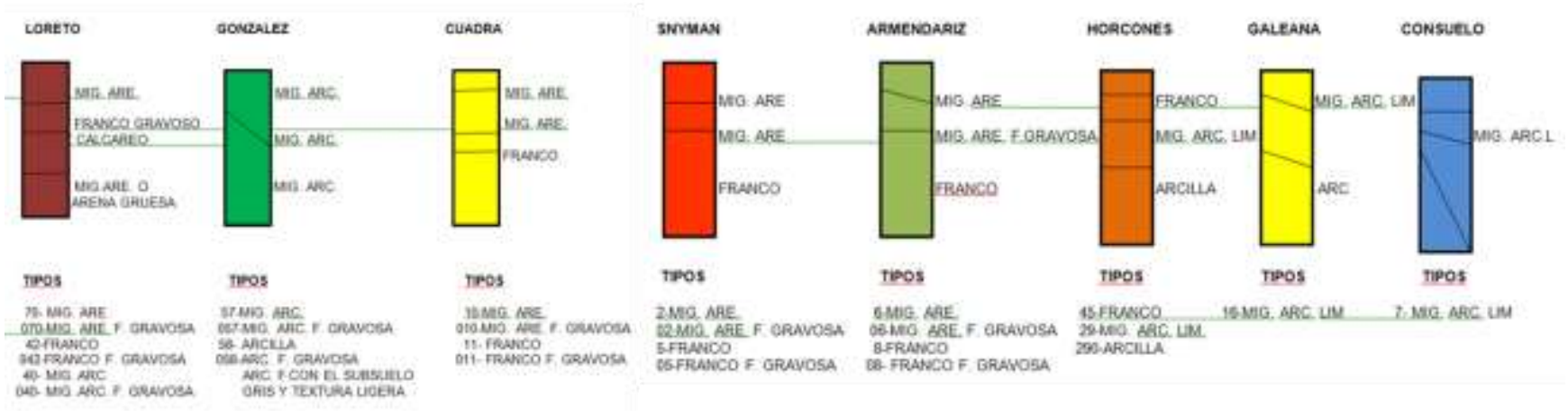

Análisis de la superficie sembrada de los principales cultivos e insecticidas usados en estos en el Distrito de Riego 005

En el Cuadro 2 se observa la superficie sembrada de los principales cultivos analizados en este estudio, en el Distrito de Riego 005 el cual cuenta con una superficie de 54096 has
(SEMARNAT, CNA，2015). Como puede observarse los cultivos que más superficie ocupan son la alfalfa, seguida con nogal y cacahuate, siendo también significativo el cultivo de maíz forrajero, ocupando los cultivos analizados una superficie de 5249 has, el resto de las 54096 has es sembrado por otros cultivos.

Cuadro 2. Superficie sembrada de los principales cultivos del Distrito de Riego 005.

\begin{tabular}{|l|c|}
\hline Cultivo & $\begin{array}{c}\text { Superficie sembrada } \\
\text { (has) }\end{array}$ \\
\hline Algodón & 3115 \\
\hline Cacahuate & 6646 \\
\hline Cebolla & 1789 \\
\hline Chile verde & 3264 \\
\hline Maíz forrajero & 5047 \\
\hline Sandia & 2761 \\
\hline Alfalfa & $\mathbf{2 1 5 7 2}$ \\
\hline Nogal & $\mathbf{8 3 0 2}$ \\
\hline
\end{tabular}

El Cuadro 3 muestra los principales insecticidas y dosis utilizadas en el control de plagas por cultivo en la región de acuerdo a la información recabada en este trabajo (Lujan et. al., 2012; INFAP, 2012; Orozco et. al., 2012; Payan, 2012; Quiñonez et. al., 2012; Tarango, 2012). 
Cuadro 3. Insecticidas usados en los principales cultivos sembrados en el Distrito de riego 005.

\begin{tabular}{|c|c|c|c|c|c|c|c|}
\hline Algodón & $\begin{array}{l}\text { Maiz } \\
\text { Forrajero }\end{array}$ & C acah uate & Alfalfa & Sandia & Ch ile jalapeño & Cebolla & Nogal \\
\hline $\begin{array}{l}\text { Thiodán } 35 \mathrm{CE} \\
\text { (Endosulfan) } \\
2.0 \mathrm{Vha}\end{array}$ & $\begin{array}{l}\text { Malatión CE } \\
1.0 \mathrm{Vha}\end{array}$ & $\begin{array}{l}\text { Folidol M-72 } \\
\text { (Paration) } \\
\text { 1. } 0 \mathrm{l} / \mathrm{ha}\end{array}$ & $\begin{array}{l}\text { Thio dán } 35 \mathrm{CE} \\
\text { (Endosulfan) } \\
2.0 \mathrm{l} / \mathrm{ha}\end{array}$ & $\begin{array}{l}\text { Lannate (M etomilo) } \\
0.4 \mathrm{~kg} / \mathrm{ha}\end{array}$ & $\begin{array}{l}\text { Diazinon } 25 \\
\text { (Basudin) } \\
1.5 \mathrm{l} / \mathrm{ha} \\
\end{array}$ & $\begin{array}{l}\text { Gusation M-20 } \\
\text { (Azinfos-Metil) } \\
2.01 / \text { ha }\end{array}$ & $\begin{array}{l}\text { Thio dán } 35 \mathrm{CE} \\
\text { (En do sulfan) } \\
1.5 \mathrm{l} / \text { ha }\end{array}$ \\
\hline $\begin{array}{l}\text { Vy dat e CLV } \\
\text { (Oxamilo) } \\
0.7 \mathrm{l} / \mathrm{ha}\end{array}$ & $\begin{array}{l}\text { Clorpirif os etil } \\
1.0 \mathrm{Vha}\end{array}$ & $\begin{array}{l}\text { Diazinon } 25 \\
\text { (Basudin) } \\
1.5 \mathrm{l} / \mathrm{ha}\end{array}$ & $\begin{array}{l}\text { Malatión } \\
2.0 \mathrm{l} / \mathrm{ha}\end{array}$ & $\begin{array}{l}\text { Sevín } 80 \mathrm{PH} \\
\text { (Carbaril) } 1.0 \mathrm{~kg} / \mathrm{ha}\end{array}$ & $\begin{array}{l}\text { Thiodán } 35 \mathrm{CE} \\
\text { (Endosulfan) } \\
1.5 \mathrm{l} / \mathrm{ha}\end{array}$ & $\begin{array}{l}\text { Diaz inon } 25 \\
\text { (Bas ud in) } \\
1.5 \mathrm{l} / \mathrm{ha}\end{array}$ & $\begin{array}{l}\text { Pirimor } \\
(50 \mathrm{~g} / 1001 \\
\text { agua/ha) }\end{array}$ \\
\hline $\begin{array}{l}\text { Folidol M-72 } \\
\text { (Paration) } \\
1.0 \mathrm{l} / \mathrm{ha}\end{array}$ & $\begin{array}{l}\text { Lannate } \\
\text { (M etomilo) } \\
0.4 \mathrm{~kg} / \mathrm{ha}\end{array}$ & $\begin{array}{l}\text { Nudrin } \\
1 \mathrm{~kg} / \mathrm{ha}\end{array}$ & 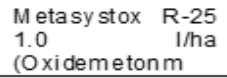 & $\begin{array}{l}\text { Thionex } 35 \% \mathrm{CE} \\
1.5 \mathrm{l} / \mathrm{ha}\end{array}$ & $\begin{array}{l}\text { Folidol M }-72 \\
\text { (Paration) } \\
1.0 \mathrm{l} / \mathrm{ha}\end{array}$ & $\begin{array}{l}\text { Folidol M-72 } \\
\text { (Paration) } \\
\text { 1. } 0 \mathrm{l} / \mathrm{ha}\end{array}$ & $\begin{array}{l}\text { Dipel } 2 X \\
\text { (Bacillus T.) } \\
1 \mathrm{~kg} / \mathrm{ha}\end{array}$ \\
\hline $\begin{array}{l}\text { Gusatión } 35 \mathrm{PH} \\
1.2 \mathrm{~kg} / \mathrm{ha}\end{array}$ & $\begin{array}{l}\text { Diazinon } 25 \\
\text { (Basudin) } \\
1.5 \mathrm{l} / \mathrm{ha} \\
\end{array}$ & $\begin{array}{l}\text { Sevín } 80 \mathrm{PH} \\
\text { (Carbaril) } \\
1.0 \mathrm{~kg} / \mathrm{ha}\end{array}$ & $\begin{array}{l}\text { Lannate } \\
\text { (M etomilo ) } \\
0.35 \mathrm{~kg} / \mathrm{ha}\end{array}$ & Aflix $1.0 \mathrm{Vha}$ & $\begin{array}{l}\text { Tamarón } 600 \\
\text { (Metami dof os) } \\
1.0 \mathrm{l} / \mathrm{ha}\end{array}$ & $\begin{array}{l}\text { Lannate LV } \\
1.5 \mathrm{l} / \mathrm{ha}\end{array}$ & $\begin{array}{l}\text { Supracid } 40 \mathrm{E} \\
\text { (Metidation) } \\
1.5 \mathrm{I} / \text { ha }\end{array}$ \\
\hline $\begin{array}{l}\text { Hostathión } 40 \\
\text { CE (triaz of om) } \\
1.5 \text { Vha }\end{array}$ & $\begin{array}{l}\text { Dimetoato } \\
1 \mathrm{I} / \mathrm{ha}\end{array}$ & $\begin{array}{l}\text { Nuvacrón } \\
\text { M onocrotofo } \\
\text { s) } 1.01 / \text { ha }\end{array}$ & $\begin{array}{l}\text { Folidol M-72 } \\
\text { (Paration me tílico) } \\
\text { 1. } 0 \text { Vha }\end{array}$ & $\begin{array}{l}\text { Permetrin } 50 \mathrm{CE} \\
\text { (A mbush) } 0.4 \mathrm{Vha}\end{array}$ & 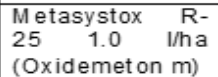 & $\begin{array}{l}\text { Karate } \\
0.4 \mathrm{l} / \mathrm{ha}\end{array}$ & $\begin{array}{l}\text { Confirm 2F } \\
600 \mathrm{mVha}\end{array}$ \\
\hline $\begin{array}{l}\text { Lannate } \\
\text { (M etomilo) } 0.4 \\
\mathrm{~kg} / \mathrm{ha}\end{array}$ & $\begin{array}{l}\text { Folimat } \\
20 \mathrm{~kg} / \mathrm{ha}\end{array}$ & $\begin{array}{l}\text { Fura dán } \mathrm{G} \\
5 \% 20 \mathrm{~kg} / \mathrm{ha} \\
\text { (Carbofuran) }\end{array}$ & $\begin{array}{l}\text { Clorpirifos etil } \\
1.0 \mathrm{l} / \mathrm{ha}\end{array}$ & $\begin{array}{l}\text { Ciromazina (Trigard } \\
75 \mathrm{PH} \text { ) } \\
0.15 \mathrm{~kg} / \mathrm{ha}\end{array}$ & $\begin{array}{l}\text { Cir omazina } \\
\text { (Trigard } 75 \mathrm{PH}) \\
0.15 \mathrm{~kg} / \mathrm{ha}\end{array}$ & $\begin{array}{ll}\text { M etasystox } R- \\
25 \quad 1.0 \quad 1 / \mathrm{ha} \\
\text { (Oxidemeton } \mathrm{m} \text { ) }\end{array}$ & \\
\hline $\begin{array}{l}\text { Supracid } 40 \mathrm{E} \\
\text { (M etidation) } \\
1.5 \mathrm{Vha}\end{array}$ & $\begin{array}{l}\text { Rogor } \mathrm{CE} \\
38.71 .0 \mathrm{l} / \mathrm{ha}\end{array}$ & & $\begin{array}{l}\text { Dipel } 2 X \\
\text { (Bacillus } \\
\text { Thuringiensis) } \\
1 \mathrm{~kg} / \mathrm{ha}\end{array}$ & $\begin{array}{l}\text { Naled } 90 \\
1.5 \mathrm{Vha}\end{array}$ & $\begin{array}{l}\text { Gusation M-20 } \\
\text { (Azinf os- M etil) } \\
2.0 \mathrm{l} / \mathrm{ha}\end{array}$ & & \\
\hline $\begin{array}{l}\text { Aflix } \\
1.0 \text { Vha } \\
\text { Hérald } 375 \\
0.5 \text { Vha } \\
\end{array}$ & $\begin{array}{l}\text { Furadán G } \\
5 \% 20 \mathrm{~kg} / \mathrm{ha} \\
\text { (Carbofuran } \\
\text { Counter G } \\
5.020 \mathrm{~kg} / \mathrm{ha} \\
\end{array}$ & & & & $\begin{array}{l}\text { Sevín } 80 \mathrm{PH} \\
\text { (Carbaril) } 2.0 \\
\mathrm{~kg} / \mathrm{ha} \\
\text { Nudrín } 90 \\
350 \mathrm{gr} / \mathrm{ha} \\
\end{array}$ & & \\
\hline $\begin{array}{l}\text { Metasystox R- } \\
251.0 \quad \text { I/ha } \\
\text { (Oxidemet on } \mathrm{m} \text { ) }\end{array}$ & $\begin{array}{l}\text { Folidol M-72 } \\
\text { (Paration) } \\
1.0 \mathrm{l} / \mathrm{ha} \\
\end{array}$ & & & & $\begin{array}{l}\text { Permetrin } 50 \mathrm{CE} \\
\text { (Ambush) } \\
0.4 \mathrm{l} / \mathrm{ha}\end{array}$ & & \\
\hline $\begin{array}{l}\text { Dipel } 2 \mathrm{X} \\
\text { (Bacillus } \\
\text { Thuringiensis) } \\
1.0 \mathrm{~kg} / \mathrm{ha} \\
\text { Kelthane MF } \\
\text { (Dicofol) } \\
2.5 \mathrm{l} / \mathrm{ha}\end{array}$ & $\begin{array}{l}\text { Sevín } 80 \mathrm{PH} \\
\text { (Carbaril) } 2.0 \\
\mathrm{~kg} / \mathrm{ha}\end{array}$ & & & & & & \\
\hline $\begin{array}{l}\text { Sevín } 80 \mathrm{PH} \\
\text { (Carbaril) } \\
2.5 \mathrm{~kg} / \mathrm{ha}\end{array}$ & & & & & & & \\
\hline $\begin{array}{l}\text { Larvín } 375 \mathrm{SA} \\
\text { (Thiodicarb) } \\
1.5 \mathrm{Vha}\end{array}$ & & & & & & & \\
\hline $\begin{array}{l}\text { Orthene } 75 \\
1 \mathrm{~kg} / \mathrm{ha} \\
\text { Tálstar } 100 \mathrm{CE} \\
0.5 \quad 1 / \mathrm{ha} \\
\text { (Bifelt rin) }\end{array}$ & & & & & & & \\
\hline $\begin{array}{lc}\text { Decís } 2.5 & \mathrm{CE} \\
0.5 & \mathrm{I} / \mathrm{ha} \\
\text { Deltametrina }\end{array}$ & & & & & & & \\
\hline
\end{tabular}

Después del análisis de los insecticidas usados en los principales cultivos que se producen en el Distrito de riego 005 se procedió al determinar el metabolismo y residuos de estos para poder determinar de manera general el posible destino y riesgo de estos en el medio ambiente. El cuadro 4 muestra un resumen producto de este análisis, resaltándose en las observaciones su relación con su movilidad y persistencia y peligro en los suelos agrícolas.

Cuadro 4. Productos fitosanitarios según su clase toxicológica analizados.

\begin{tabular}{|l|l|c|c|}
\hline \multicolumn{4}{|c|}{ Productos Fitosanitarios según clase toxicológica } \\
\hline Principio activo & Grupo químico & $\begin{array}{c}\text { Clasificación del } \\
\text { peligro }\end{array}$ & Observaciones \\
\hline Sevin (Carbaril) & Carbamato & Muy Toxico & Vida media de 7 d en suelos aerobios y 28 d en \\
& & & anaerobios \\
\hline
\end{tabular}


Moncayo-Cassiano et al., 2017

\begin{tabular}{|c|c|c|c|}
\hline Naled & Órganofosforado & Muy Toxico & Degradación rápida $(<8 \mathrm{~h})$ \\
\hline Malatión & Organofosforado & Muy Toxico & $\begin{array}{l}\text { Muy móvil en suelos limosos y areno } \\
\text { limosos }\end{array}$ \\
\hline $\begin{array}{l}\text { Folidol M } \\
\text { (Paration } \\
\text { Metil) }\end{array}$ & Organofosforado & Muy Toxico & $\begin{array}{l}\text { Degradación rápida. Movilidad moderada, } \\
\text { no se bioacumula }\end{array}$ \\
\hline $\begin{array}{l}\text { Vydate } \\
\text { (Oxamil) }\end{array}$ & Carbamato & Muy Toxico & $\begin{array}{c}\text { Degradación rápida } \\
\text { (1 sem) móvil en el suelo, puede lixiviarse }\end{array}$ \\
\hline $\begin{array}{l}\text { Gusathion A } \\
\text { (Azinfos- etil) }\end{array}$ & Organofosforado & Muy Toxico & $\begin{array}{l}\text { Desaparición lenta de residuos en frutos } \\
\text { almacenados }\end{array}$ \\
\hline $\begin{array}{l}\text { Supracid } \\
\text { (Metidation) }\end{array}$ & Organofosforado & Muy Toxico & $\begin{array}{c}\text { Degradación rápida (5 a } 23 \text { d) y poca } \\
\text { movilidad }\end{array}$ \\
\hline Isofenfos & Organofosforado & Muy Toxico & Degradación no rápida en suelos y plantas \\
\hline $\begin{array}{l}\text { Etoprofos } \\
\text { (Mocap) }\end{array}$ & Organofosforado & Muy Toxico & $\begin{array}{l}\text { Vida media depende de textura y pH (14 a } \\
\qquad 87 \mathrm{~d})\end{array}$ \\
\hline $\begin{array}{l}\text { Terbufos } \\
\text { (Counter) }\end{array}$ & Organofosforado & Muy Toxico & Descomposición rápida. No se bioacumula \\
\hline $\begin{array}{l}\text { Nuvacron, } \\
\text { Azodrin } \\
\text { (Monocrotofo } \\
\text { s) }\end{array}$ & Organofosforado & Muy Toxico & $\begin{array}{c}\text { Vida media en suelo: } 4 \text { a } 7 \text { d, puede alcanzar } \\
\text { acuíferos }\end{array}$ \\
\hline $\begin{array}{l}\text { Lannate } \\
\text { (Metomilo) }\end{array}$ & Carbamato & Toxico & $\begin{array}{l}\text { En condiciones anaerobias se degrada } \\
\text { rápidamente }\end{array}$ \\
\hline $\begin{array}{l}\text { Thiodan } \\
\text { (Endosulfan) }\end{array}$ & Fosfórico & Toxico & Se degrada lentamente \\
\hline $\begin{array}{l}\text { Metamidofos } \\
\text { (Tamaron) }\end{array}$ & Organofosforado & Toxico & $\begin{array}{l}\text { Vida media en suelo } 2 \mathrm{~d} \text {. y en agua de } 5 \text { a } 27 \\
\text { d }\end{array}$ \\
\hline $\begin{array}{l}\text { Furadan } \\
\text { (Carbofuran) }\end{array}$ & Carbamato & Toxico & $\begin{array}{l}\text { Se absorbe fuertemente en el suelo hasta su } \\
\text { degradación microbial }\end{array}$ \\
\hline $\begin{array}{l}\text { Gusathion M } \\
\text { ( Azinfos- } \\
\text { Metil) }\end{array}$ & Organofosforado & Toxico & $\begin{array}{l}\text { Vida media en suelo aerobio: } 21 \mathrm{~d} \text {. y } 68 \text { en } \\
\text { anaerobio }\end{array}$ \\
\hline Forato & Organofosforado & Toxico & $\begin{array}{l}\text { Móvil a muy móvil de acuerdo al suelo. } \\
\text { Algunos de sus compuestos son muy } \\
\text { lixiviables. }\end{array}$ \\
\hline $\begin{array}{l}\text { Metasystox R } \\
\text { (Oxidemeton } \\
\text { metil) }\end{array}$ & Organofosforado & Toxico & $\begin{array}{c}\text { Se degrada rápidamente en el suelo y es muy } \\
\text { móvil }\end{array}$ \\
\hline $\begin{array}{l}\text { Hostahtion } 40 \\
\text { CE } \\
\text { (Triazofos) }\end{array}$ & Organofosforado & Toxico & $\begin{array}{l}\text { Vida media en suelos aerobios 6-12 d y en } \\
\text { agua }<3 \mathrm{~d} \text {. }\end{array}$ \\
\hline $\begin{array}{l}\text { Larvin } \\
\text { (Tiodicarb) }\end{array}$ & Carbamato & Toxico & $\begin{array}{l}\text { Biodegradación rápida ( } 3-8 \mathrm{~d}) \text { movilidad } \\
\text { baja y no es persistente en el ambiente }\end{array}$ \\
\hline
\end{tabular}


Moncayo-Cassiano et al., 2017

\begin{tabular}{|c|c|c|c|}
\hline $\begin{array}{l}\text { Ometoato } \\
\text { (Folimat) }\end{array}$ & Organofosforado & Toxico & $\begin{array}{c}\text { muy móvil en suelos y vida media de pocos } \\
\text { días }\end{array}$ \\
\hline $\begin{array}{l}\text { Permetrin } \\
\text { (Ambush) }\end{array}$ & $\begin{array}{l}\text { Piretroide } \\
\text { sintético }\end{array}$ & Nocivo & $\begin{array}{l}\text { Degradación rápida en suelo y agua. } \\
\text { Fotoestable }\end{array}$ \\
\hline $\begin{array}{l}\text { Dimetoato } \\
\text { (Rogor) }\end{array}$ & Organofosforado & Nocivo & $\begin{array}{l}\text { Vida media 2-4 d. En plantas } \\
\text { moderadamente persistente }\end{array}$ \\
\hline $\begin{array}{l}\text { Lambda } \\
\text { Cihalotrin } \\
\text { (Karate) }\end{array}$ & $\begin{array}{l}\text { Piretroide } \\
\text { Sintético }\end{array}$ & Nocivo & Amplio espectro \\
\hline Foxim & Organofosforado & Nocivo & $\begin{array}{l}\text { Vida media } 2 \text { a } 3 \text { meses. Acción residual y } \\
\text { movilidad en el suelo. }\end{array}$ \\
\hline Fipronil & Fenil Pirazoles & Nocivo & $\begin{array}{l}\text { Absorción directamente proporcional al } \\
\text { contenido de materia orgánica }\end{array}$ \\
\hline $\begin{array}{l}\text { Clorpirifos } \\
\text { (Dursban) }\end{array}$ & Organofosforado & Nocivo & $\begin{array}{l}\text { Degradación lenta (92-341 d. en suelos } \\
\text { ácidos y 11-200 d en alcalinos) }\end{array}$ \\
\hline $\begin{array}{l}\text { Diazinon } \\
\text { (Basudin) }\end{array}$ & Organofosforado & Nocivo & Relativamente persistente ( más de $50 \mathrm{~d}$ ) \\
\hline $\begin{array}{l}\text { Teflutrin } \\
\text { (Force) }\end{array}$ & $\begin{array}{l}\text { Piretroide } \\
\text { Sintético }\end{array}$ & Nocivo & Degradación rápida y poca movilidad \\
\hline $\begin{array}{l}\text { Herald } \\
\text { (Fenpropatrin) }\end{array}$ & $\begin{array}{l}\text { Piretroide } \\
\text { Sintético }\end{array}$ & Nocivo & $\begin{array}{c}\text { Duración en suelo 1-5 d. En agua de rio 2-7 } \\
\text { sem. }\end{array}$ \\
\hline $\begin{array}{l}\text { Kelthane } \\
\text { (Dicofol) }\end{array}$ & Organofosforado & Nocivo & $\begin{array}{c}\text { Estable en el suelo casi } 1 \text { año con } \\
\text { persistencia hasta } 4 \text { años y bioacumulable }\end{array}$ \\
\hline $\begin{array}{l}\text { Talstar } \\
\text { (Bifentrin) }\end{array}$ & $\begin{array}{l}\text { Piretroide } \\
\text { Sintético }\end{array}$ & Nocivo & $\begin{array}{l}\text { Vida media 7-62 d. Poca movilidad en } \\
\text { suelos arenosos e inmóvil en arcillosos }\end{array}$ \\
\hline $\begin{array}{l}\text { Pirimicarb } \\
\text { (Pirimor) }\end{array}$ & $\begin{array}{c}\text { Carbamat } \\
\text { o }\end{array}$ & Nocivo & $\begin{array}{c}\text { Vida media de } 7 \text { a } 234 \text { d según tipo de suelo. } \\
\text { Puede lixiviarse }\end{array}$ \\
\hline $\begin{array}{l}\text { Decis } \\
\text { (Deltametrin) }\end{array}$ & $\begin{array}{l}\text { Piretroide } \\
\text { Sintético }\end{array}$ & Nocivo & $\begin{array}{l}\text { Vida media } 12-\mathrm{a} 50 \mathrm{~d} \text {. Poco soluble en agua. } \\
\text { Se fija en } \operatorname{los} 2-2.5 \mathrm{~cm} \text {. Alta retención en la } \\
\text { MO del suelo }\end{array}$ \\
\hline $\begin{array}{l}\text { Ciromazina } \\
\text { (Trigard) }\end{array}$ & & Cuidado & $\begin{array}{l}\text { Vida media en suelo arcillo arenoso } 139 \mathrm{~d} \text { y } \\
\text { en arenoso } 116 \mathrm{~d} \text {. No contamina ambiente }\end{array}$ \\
\hline $\begin{array}{l}\text { Orthene } \\
\text { (Acefato) }\end{array}$ & Benzoil Hidracina & Cuidado & Vida media 3-6 d. Se lixivia fácilmente \\
\hline $\begin{array}{l}\text { Tebufenocida } \\
\text { (Confirm) }\end{array}$ & Benzoil Hidracina & Bajo & $\begin{array}{c}\text { Vida media entre } 7 \text { y } 105 \text { d según suelo. } \\
\text { Poca movilidad y riesgo de contaminación } \\
\text { de aguas }\end{array}$ \\
\hline Aflix & Fosfórico & Bajo & Se deben tener cuidados con su aplicación \\
\hline $\begin{array}{l}\text { Bacillus } \\
\text { Thuringiensis } \\
\text { (Dipel) }\end{array}$ & Biológico & Bajo & $\begin{array}{l}\text { Degradación rápida en luz UV a compuestos } \\
\text { no tóxicos }\end{array}$ \\
\hline
\end{tabular}


La información anterior muestra que los principales insecticidas usados en la Región Centro-Sur del estado de Chihuahua son esencialmente organofosforados de los cuales en su mayoría son considerados muy tóxicos y el resto tóxicos y nocivos. El análisis del metabolismo y residuos muestra que los principales insecticidas que son muy móviles y que por lo tanto lixiviables pueden alcanzar los acuíferos en los suelos arenosos de la región son el Malation usado en alfalfa y maíz forrajero, Vydate usado en algodón, Nuvacron en cacahuate y Pirimor en nogal, por lo cual no es recomendable su aplicación en este tipo de suelos.

De acuerdo a este mismo análisis, se puede observar que dos de los insecticidas podrían acumularse en los suelos; el Endosulfan ya que se degrada muy lentamente y el Furadan que se absorbe fuertemente en los suelos. Lo anterior es de tomar en cuenta puesto que el Endosulfan se aplica en alfalfa, nogal, chile jalapeño y algodón que son los cultivos de mayor superficie sembrada (Alrededor del $67 \%$ ) y podría persistir en los suelos de acuerdo a su uso, así como lo haría el Furadan usado principalmente en cacahuate y maíz forrajero por lo cual se recomendaría precaución en su uso.

Por último, se puede mencionar que es difícil cuantificar la cantidad de pesticidas aplicados en los cultivos, puesto que se cuentan con diferentes alternativas para el control de plagas, sin embargo, muchos de los pesticidas usados en el Distrito de Riego 005 no son persistentes en el ambiente, y ninguno se encuentran en la lista de plaguicidas prohibidos por la COFEPRIS (2016). Sin embargo, su uso intensivo es lo que puede ocasionar peligros ambientales a corto y largo plazo.

\section{CONCLUSIÓN}

Las sustancias contaminantes como los plaguicidas se dispersan y transportan sobre y dentro de los recursos naturales. La contaminación ambiental es causada por el uso excesivo y continuo de los plaguicidas, y comienza cuando estos compuestos entran en el ambiente, lo que supone la existencia de potenciales efectos nocivos para el hombre, la fauna en general y la vegetación.

Se observa que muchos de los pesticidas analizados son aplicados en grandes cantidades en la Región Centro-Sur del Estado de Chihuahua, debido a su eficacia y bajo costo.

Las características de algunos de los suelos del Distrito de Riego 005 pueden estar contribuyendo a que algunos pesticidas alcancen cuerpos de agua.

Se recomienda tomar en cuenta los pesticidas que tienden a degradarse lentamente o absorberse en el suelo pues esto puede ocasionar su acumulación en este.

La adición de materia orgánica puede ser un factor que contribuya a la degradación de los plaguicidas así como a su retención en el suelo de tal manera que se evite su transporte hacia los mantos acuíferos y cuerpos de agua superficiales.

Las interacciones entre los plaguicidas aplicados y el ambiente dependen fundamentalmente de las propiedades físicas y químicas de estos así como de los factores ambientales, por lo cual es importante que los productores consideren el tipo de plaguicida a aplicar, características del suelo, dosis recomendada y sobre todo tener en cuenta que las buenas prácticas de manejo de estos y la adición de materia orgánica pueden reducir los riesgos hacia el ambiente.

\section{LITERATURA CITADA:}

Arias-Esteves M., López-Periago E., Martínez Carballo E., Simal-Gandara j., Mejuto J.C y García-Río L. 2008. The movility and degradation of pesticides in soils and the pollution of groundwater resources. Agriculture, Ecosystems and Environment journal. 123 (2008) 247-260.

https://doi.org/10.1016/j.agee.2007.07.011

Carvalho, F. P.; D. D. Nhan; C. Zhong; T. Tarares; S. Klaine: 1998. Tracking

Revista Científica Biológico Agropecuaria Tuxpan 5 (2) 
Pesticides in the Tropics, Bulletin IAEA40 (3): 2430.

CNA. Comisión Nacional del Agua. 2013. Oficina Delicias Chihuahua.

COFEPRIS (Comisión Federal para la Protección contra Riesgos Sanitarios). 2016. Catálogo de plaguicidas.

De Liñán V. C. 2003. Farmacología Vegetal. Edición Agrotecnica S.S.L. 3 ${ }^{\text {a }}$. Edición.

Dokic, M., Bilandzic, N., Briski, F. 2012. Procedures for removal of pesticides from the environment. (http://web.ebscohost.com/ehost/delibery)

Fenoll J., Ruiz E., Flores P., Hellin P., Navarro S. 2011. Reduction of the movement and persistence of pesticides in soil through common agronomic practices. https://doi.org/10.1016/j.chemosphere.2011.0

INIFAP. 2012. Técnicas para la producción de cebolla en la región de Delicias, Chih. INIFAP (Instituto Nacional de Investigaciones Forestales y Agropecuarias).

Luján F.M., Acosta R. G., Quiñonez P. F.J., Uribe M. R., Berzoza M. M., Aldaba M. J.L., Galván L. R., Rodríguez M. R., Chávez S. N. 2012. Chile jalapeño. INIFAP.

Mosquera V., C. E. 2010. Evaluación del movimiento del agua y plaguicidas en suelos de uso agrícola y su predicción de contaminación hacia aguas subterráneas. Universidad Nacional de Colombia.
OPS (Organización panamericana de la salud). 2012. Clasificación toxicológica de los plaguicidas. ops.org.ar/publicaciones/publicaciones virtuales/proyectoPlaguicidas.pdf.

Orozco H.G., Quiñonez P. F. y Aldaba M. J.L. 2012. Como cultivar cacahuate en Delicias Chih. INIFAP. Campo Experimental "Delicias".

Orozco (a), H.G., Uribe, M.H., Quiñonez, P. F.J. 2012. Guía para la asistencia técnica agrícola. Maíz de riego. INIFAP.

Ortiz-Hernández, Ma. L., \& Sánchez-Salinas, E. 2010. Biodegradation of the organophosphate pesticide tetrachlorvinphos by bacteria isolated from agricultural soils in México. Revista internacional de contaminación ambiental, 26(1), 27-38.

Payán G. J.A. 2012. Técnica para producir alfalfa en la región de Delicias, Chih. INIFAP.

Quiñones P. F.J., Galván L. R. y Báez I. F. 2012. Tecnología de producción de algodón en la región Centro-Sur del estado de Chihuahua. INIFAP.

Rodriguez C. M.S., Jones J.E. and Bending G.D. 2006. Field-scale study of the variability in pesticide biodegradation with soil depth and its relationship with soil characteristics. Elsevier. Journal of Soil Biology\&Biochemistry 38 (2006) 2910-2918. https://doi.org/10.1016/j.soilbio.2006.04.051

SEMARNAT, CNA. 2015. Estadísticas agrícolas de los distritos de riego. Año agrícola 2013-2014. 2015.

Tarango R. S.H. 2012. Nogal pecanero INIFAP 
Copyright (c) 2017 Vicente Moncayo Cassiano, Bertha Alicia Rivas Lucero, Sergio Guerrero Morales,

Gabriel Zúñiga Avila1 y José E duar do Magaña Magaña

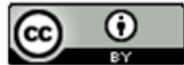

Este tex to está protegido por una licencia licencia Creative Commons $\underline{4.0}$

Usted es libre para Compartir — copiar y redistribuir el material en cualquier medio o form ato- y Adaptar el documento -remezdar, transformar y crear a partir del material- para cualquier propósito,, incluso para fines comerciales, siempre que cumpla la condición de:

Atribución: Usted debe dar crédito a la obra original de manera adecuada, proporcionar un enlace a la licencia, e in dicar si se han realizado cam bios. Puede hacerlo en cualquier form a razonable, pero no de form a tal que sugiera que tiene el apoyo del licenciante o $1 \circ$ recibe por el uso que hace de la obra.

Resumendelicencia - Textocompletodelalicencia 